Volume 8, No. 7, July - August 2017

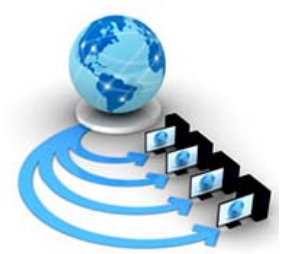

International Journal of Advanced Research in Computer Science

REVIEW ARTICLE

\author{
Available Online at www.ijarcs.info
}

\title{
A STUDY OF REMOTE SENSING USING IMAGE FUSION: A REVIEW
}

\author{
Ishpreet Kaur \\ Department of Computer Engineering, \\ Punjabi University, Patiala \\ Punjab, INDIA
}

\author{
Nirvair Neeru \\ Department of Computer Engineering, \\ Punjabi University, Patiala \\ Punjab, INDIA
}

\begin{abstract}
Remote sensing is an approach to study remote area via images. But the limitation of remote sensed images is that they lack in giving complete information of the area. Therefore, the remote sensing is combined with image fusion by virtue of which the images are combined to get the hidden information. The image fusion is a technique that extracts relevant information from two or more source images of same scenes into single image. There is need of image fusion, for high resolution on panchromatic and multispectral images or real world images for better vision. The aim of image fusion (IF) is to coordinate complementary multi-temporal and multisensory data into one new image containing information, the quality of which cannot be achieved otherwise. The keyword quality definition and measurement is based on the specific applications. This paper provides a literature review on the techniques of image fusion like simple average, maximum, Principal Component Analysis (PCA), Discrete Wavelet Transform (DWT), etc. Image fusion is done to get a more enhanced and informative and more quality image from two or more images that are taken from completely different views and sensing element. The application areas of image fusion include both military and domestic functions and even medical functions.
\end{abstract}

Keywords: Remote sensing; fusion; DWT; IHS, ICA

\section{INTRODUCTION}

The term "Remote" means the art and science of acquiring information related to an object without contacting physically to sensor and the object. Remote Sensing is a powerful approach for using a large volume of data from multi-sensor images. The effect of using the remote sensing image fusing approach is to upgrade the visual interpretation. Earth satellites like IKONOS, SPOT, Quickbird produces low quality resolution Panchromatic and multi spectral (MS) images by using spectral band combinations, the color information in a remote sensed image can be increases for a given spatial resolution. different spots in a single band seems to be similar which is complicated while differentiate them. Multiple bands can be obtained by a single multi-spectral sensor at different frequencies. Generally the sensors with having high spatial resolution, featured by occupying the radiance from different land areas where we don't have an adequate spatial resolution[4].

Geometric characterstics can easily be identified on a high spatial resolution PAN image. The adequacy of the images can be upgraded if the features of both high spectral and high spatial resolution combined into one single image. The detailed specifications of such an merged image can easily be identified and will be useful to many applications. By using convenient approach we can merge multispectral and panchromatic bands and provides a synthetic image with their best features. the process of combing this is known as multisensor merging [3]. It focuses on the combining process of spatial contents of higher resolution pan image and the color information of lower resolution multispectral image to produce good quality. The resulted fused image is a new image which is more useful for human and machine perception in an any of the image processing tasks. The hybrid quality must contain the most possible spatial information. High frequency components gives the detailed spatial information of panchromatic image while the low frequency components gives the spectral information of Multispectral image. if the components of multispectral image (high frequency components) is replaced by the panchromatic image components, the spatial resolution is enhanced but the spectral information will be lost from the high frequency components. To obtain good quality images, few conditions must be contain during fusion process. The Multispectral and panchromatic images should obtain at nearby time while taking it several changes may occur: different lightening conditions. the spectral range of multispectral must be covered by the spectral range of panchromatic image to observe the color on an image and also helps in avoiding the color distortion in the fused image.

In the fused image the replaced spectral bands components of high resolution must be similar to that of the earlier low resolution component also must match contrast to reduce artifacts. In summary the image fusion helps in combining the features of two images that are captured from remote area. It produces good quality images by virtue any user can easily view the hidden objects or things in order to take timely preventive measurements. The approach is very much beneficial to military agencies as well as for monitoring remote areas. The paper focuses on various image fusion methods for remote sensing and are discussed in next sections.

The organization of paper is as follows. Section II discusses the image fusion techniques/ methodologies. The pros and cons of the existing techniques are shown in Section III. Section IV provides the comparison among the techniques 
and the researchers efforts based on these techniques. Finally, the main conclusion as are drawn in Section V.

\section{IMAGE FUSION TECHNIQUES}

To develop this technique of remote sensing for these images is known as Image fusion. Image fusion is the technique of extracting useful and combining the relevant information from a different set of images of the same scene into a single image that plays a vital role in understanding the newly formed pixels. the resultant fused image will contain a more useful, clear, noise free and complete information of all the input images.

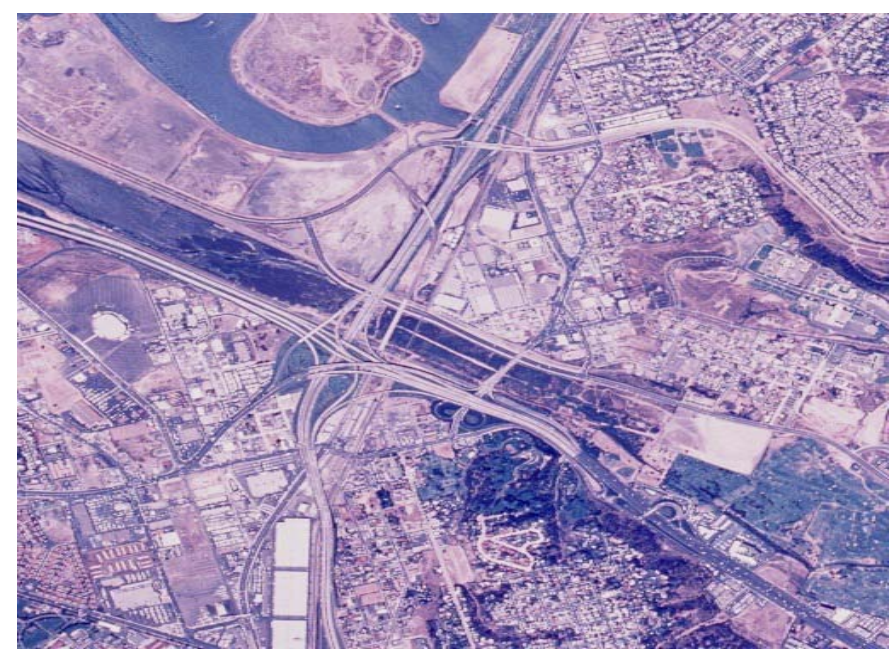

Fig. 1. An aerial remote sensed satellite image[1]

The input images can be in following ways:

- Multitemoral fusion: Data is being achieved by the identical sensor captures the same scene at different dates.

- Multifrequency fusion: Data is being achieved by the identical sensor implementing in different spectral bands.

- Multimodal fusion: Data is being identical by the images of different modalities.

- Multiview fusion: Data is being achieved by the images of same modality taken at the same time but from different locations.

- Multisensor fusion: Data is being obtained by different sensors

The benefits of using image fusion is that it helps in easy interpretation and best for identification and recognition. It sharpens the images and also to improve geometric corrections. Resulted image will be true in color and will have more resolution (improves spatial resolution) and changes are detected by using multi-temporal data, eliminates the missing information. In fog image fusion provides improved fused images. Due to its more contrast it enhances the image more. Main objective is to reduce the data storage and data transmission.

The basic problem of image fusion is to identifying the optimum method for merging the several input images. Many different fusion techniques are there to integrate a Pan image and a MS image into a multispectral image with high spatial and spectral resolution simultaneously. An ideal image fusion technique must contain three important terms, i.e. high computational efficiency, preserving high spatial resolution and reducing color distortion[9]. The vital information contained by the fused image is used in many applications such as Aerial and Satellite imaging, Medical imaging, Robot Vision, Multi-focus image fusion, Digital camera application etc. Image Fusion must follow two basic concepts. First, fused image must traverse all possible vital information, contained in the source images; second, fusion process should not introduce any artifact, noise or unuseful specification in the fused image.

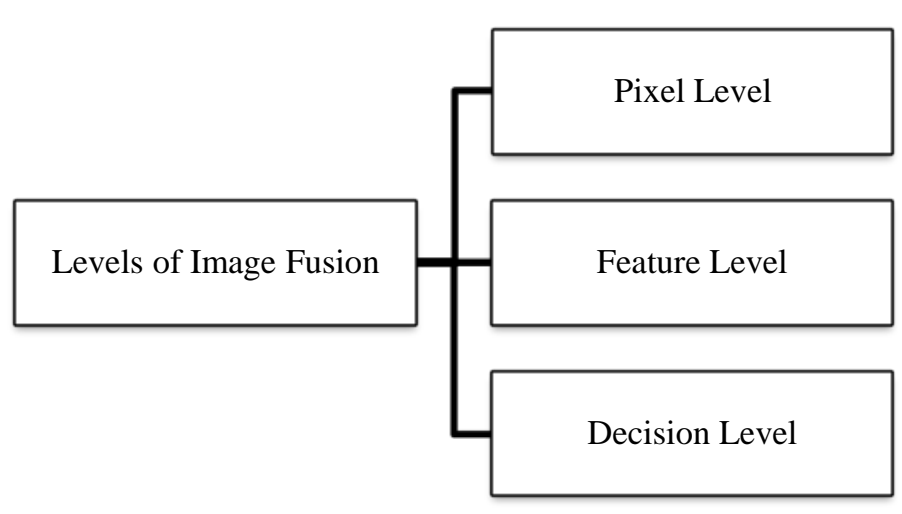

Fig. 2 Implementing Levels of Image Fusion

\section{Pixel based}

Pixel level fusion deals with information associated with each pixel and resultant image can be acquired from the correlate pixel values of source images. Pixel level fusion methods are easy to implement and provides original information in the fused image as there is minor loss of information. paramount of information to be processed, so the processing speed is slow.

\section{Feature based}

Feature level fusion, input images are get separated into regions and features (like intensities of pixels, shapes, edges or texture) and these features are used for fusion. Features that are extracted from the different source images gets collected to form an optimum feature set and further preprocessed using different schemes to form a decision[16].

\section{Decision based}

Decision level fusion is a high level fusion depends upon decisions coming from various fusing sensors. Decision level fusion methods are based on some statistics, voting, fuzzy logic, prediction and heuristics etc. In this method images are computed severely. The computed data is then refined by merging the data acquired from different sources. 


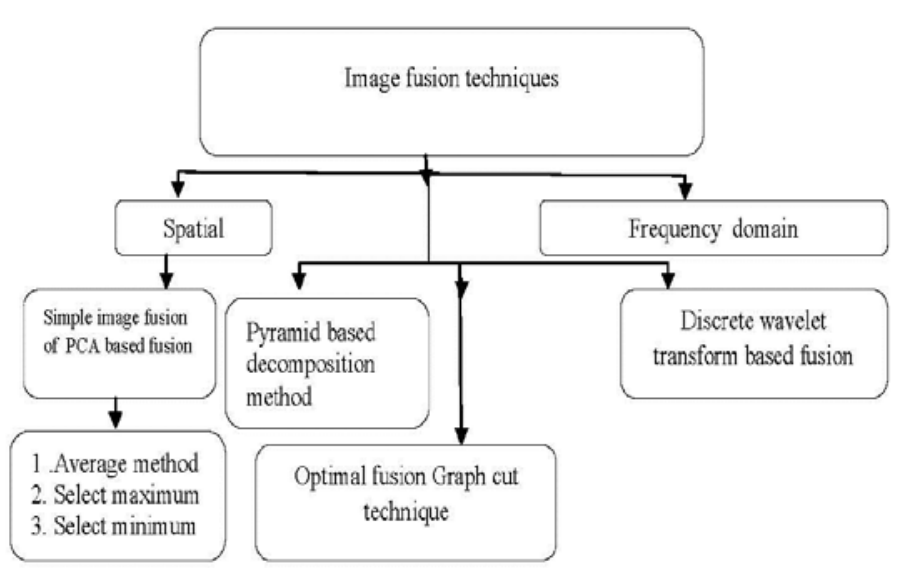

Fig.3 Classification of Image fusion Techniques

A. There are two main types of image fusion methods:

I. Spatial domain Fusion: Spatial domain technique directly deals with the pixels of origin graphics. It fuses entire graphics utilizing local spatial features such as gradient.[14]

Spatial domain fusion techniques

\section{- Average Method}

Average method focuses on all the regions of the images of higher pixel level as compare to the other regions of the images.The averages one of simplest method for suppressing any noise in the source image with major side effect of average is that it reduced contrast.To obtain an output image in which all regions are in focus, average method is used. Sum of values of pixels $(x, y)$,and then get divided by the total number of input images. Mathematically can be written as:

$$
T(x, y)=[R(x, y)+S(x, y)] / 2(I)
$$

where $\mathrm{R}(\mathrm{x}, \mathrm{y})$ and $\mathrm{S}(\mathrm{x}, \mathrm{y})$ are two source images.

\section{- Maximum method}

This algorithm takes the greatest value of each pixel such as $\mathrm{T}(\mathrm{x}, \mathrm{y})$ from source images and compare to each others. The greatest pixel value is then assigned to the corresponding pixel of new image. It is one of the straight forward and fastest algorithms but also alter the spectral information of the source image.

$T(x, y)=\sum \sum \max . R(x, y) S(x, y) m x=0, y=0(I I)$

\section{- Minimum method}

In this method, the resulatant fused image is acquired by performing the algorithm of selecting the minimun intensity of corresponding pixels values of input images.

$T(x, y)=\sum \sum \min \cdot R(x, y) S(x, y) m x=0, y=0(I I I)$

\section{- IHS Transform}

IHS (Intensity, Hue, Saturation) fusion converts a color image from the $R G B$ (Red, Green, Blue) into the IHS color space.IHS need to be controlled because in it all the data is spectral.IHS transform is used on the low spatial resolution images, then the intensity ingredient is then replaced by the high spatial resolution images. Reverse IHS is applied on new sets to form fused image. Technique used in fusion method for sharpening.
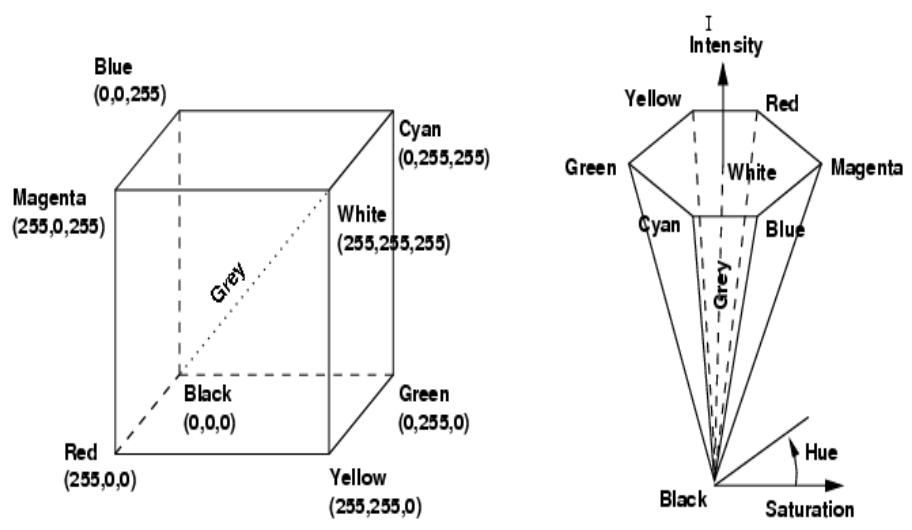

Fig.4 RGB to IHS Color Transformation [12]

\section{- Principal Component Analysis (PCA)}

The principle component analysis considers pixels from all images at its pixel location, it adds a specialized weight factor in each pixel and then takes the average of the weighted pixel. This newly generated pixel produces the fused images with same size. The PCA determines the weighted factor optimally. This technique is used in Various applications like encoding, compression, enhancement and recognition in images. It is useful in transforming multi variate datasets into newly uncorrelated linear datasets of original variables. the generated pixels are orthogonal in nature and decreases the redundant data[3].

Applications: Image classification and image compression.

\section{- Independent Component Analysis (ICA)}

Independent Component Analysis uses aggregate method for severance a multivariate signal into sub modules assuming the analytical independence of the non Gaussian signals. The appraisal of the data model of this technique is usually performed by phrasing an objective function and then minimizing or maximizing it. hence the assets of Independent component analysis method depends on twain objective function and the optimization algo[2]. Abeit the momentous Independent component contains the useful content of color image, the spatial and spectral information of the color image is not get dissevered, still a part of information is located on other ICs. So this method based seems to have fused image with high spatial content but also may contains to spectral distortion in some regions. To reduce this effect of spatial distortion we use Intensity Hue Saturation transform technique to further process the fused outcome image and reestablish its color components with that of original Ms image[2].

The Topographical ICA based method offers a more accurate directional selectivity, thus capturing the salient features of the image more accurately. Various benefits of image fusion: temporal coverage and wider spatial, reduced uncertainty, improved reliability, and increased robustness of system performance. 
the fused image is obtained.

\section{- Brovery Transform (BT)}

Brovery Transform technique was introduced by Bob Brovery also known as color normalization transform, as it based on Red, Green, Blue (RGB) color transformation and concept of intensity modulation. Its purpose is to normalize the three multispectral bands for RGB display. The mathematical procedure of the Brovery Transform (BT) can be represented as a integration of panchromatic (Pan) and multispectural (MS) images. Every MultiSpectral image is get multiplied by a ratio of the Pan image divided by the sum of the MS images. The fused R, G, B images are defined by the following equations.[11]

$R x=\frac{\mathrm{R} 1}{R 1+G 1+B 1} \times P A N(I I I)$

$G x=\frac{G 1}{R 1+G 1+B 1} \times P A N(I V)$

$B x=\frac{\mathrm{B} 1}{R 1+G 1+B 1} \times P A N(V)$

The resultant image has both high spatial and spectral resolution that is require in many remote sensing application. Thus it is the simplest method that it increases the contrast in low and high end of the image histogram for integrating data from different sensors, with the limitation that only three bands are involved.

II. Temporal Domain Fusion: In Temporal domain image is first converted into transform domain such as Fourier transform of the image is done.

Temporal domain fusion techniques

\section{- Discrete Cosine Transform (DCT)}

Discrete cosine Transform plays vital role for the compressed images in the form of MPEG, JVT etc. Discrete cosine transform method helps to convert the spatial domain image into the frequency domain image. The image can be divided into three parts as low frequency, medium frequency and high frequency. Average illumination is shown by the DC values. AC values are high frequency coefficients. The $R, G, B$ image is portioned into the blocks of size of $8 * 8$ pixels. The image is then grouped by the matrices of red, green and blue and transformed to the greyscale image.

The 2 Dimensional Discrete Cosine Transform is then applied on the greyscale image. The grayscale block frequency is transmitted from the spatial to frequency domain. Once the DCT coefficients are calculated, fused DCT coefficients are obtained by applying the fusion rule. By taking inverse DCT,
DCT based methods are more reliable in terms of time and hence they are useful in real time systems. DCT coefficients show energy compactness because all DCT coefficients are brought together in the low frequency zone. It results real output when the real-time input data is provided as an input [7].

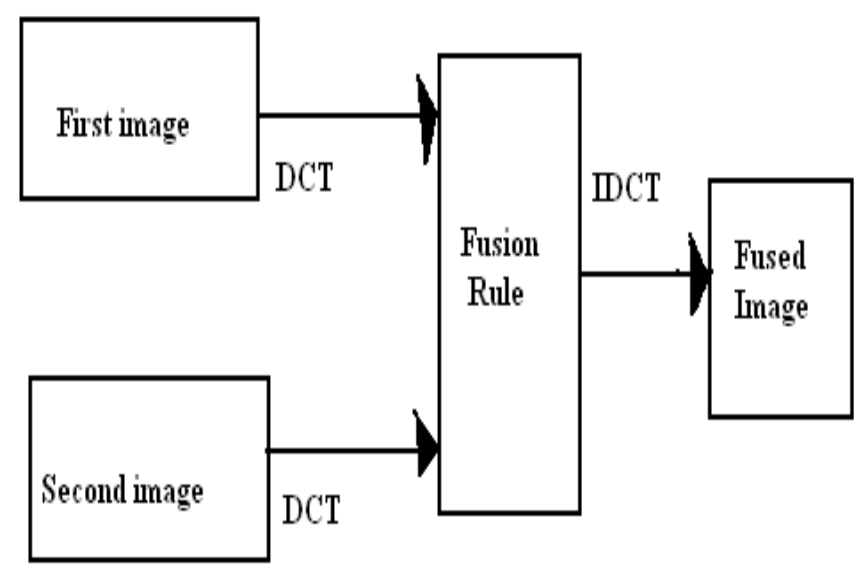

Fig. 5 Fusion with (DCT)

\section{- $\quad$ Discrete Wavelet Transform (DWT)}

In DWT, two types of filters are applied on the available frequency. These two types of filters are low pass filter and high pass filter. These filters divide the available frequency in exactly two halves. And process is applied onto each row, then to each column and collect their own frequencies from the available frequency. So, the filtering process results into two dimensional array of coefficients. This array consists of four bands low-low, high-low, low-high and high-high. Low-low band be further decompose into four bands to attain its second level decomposition. The low-low band is used for decomposition as it is easy to process. Moreover, the low-low filter has highest importance as compare to all other bands. The following figure shows three level view DWT.

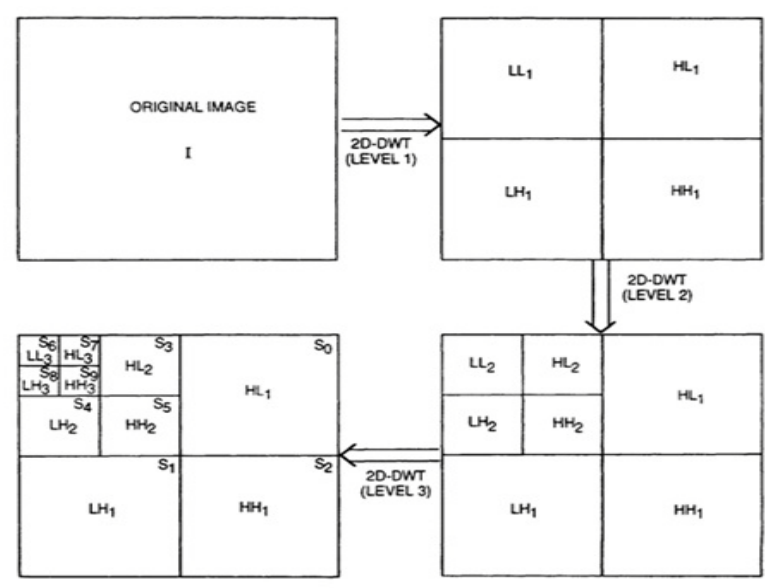

Fig. 6 Three level view of $D W T$

The DWT provides the good quality images to know the unknown or newly formed pixels and for enhancing images using interpolation. This technique includes very low and high 
frequency bands. The low frequency bands can be easily

\section{COMPARISON OF IMAGE FUSION TECHNIQUES}

The comparison of various existing image fusion techniques are discussed with its application areas, advantages and discarded without any loss of quality[9].

disadvantages. The various techniques are Brovery Transform $(B T)$, Principle Component Analysis (PCA), Intensity Hue Saturation (IHS), Independent Component Analysis (ICA), Average Method, Maximum Method, Minimum Method, Discrete Wavelet Fusion (DWT).

TABLE I. Comparison of Existing Image Fusion Techniques

\begin{tabular}{|c|c|c|c|c|}
\hline S.No & Technique & Application & Advantages & Disadvantages \\
\hline 1. & $\begin{array}{l}\text { BT(Brovery } \\
\text { Transform)[11] }\end{array}$ & $\begin{array}{l}\text { - Use to } \\
\text { LANDSATTM and SAmbine } \\
\text { images. } \\
\text { - Successful for SPOT pan } \\
\text { fusion. }\end{array}$ & $\begin{array}{l}\text { - Good to produce } R G B \text { images } \\
\text { with higher degree of contrast. }\end{array}$ & $\begin{array}{l}\text { - High contrast pixel values } \\
\text { in input. } \\
\text { - Image are depressed in } \\
\text { value in fused image. }\end{array}$ \\
\hline 2. & $\begin{array}{l}P C A \\
\text { (Principle } \\
\text { component } \\
\text { analysis)[12] }\end{array}$ & $\begin{array}{l}\text { - Face Recognition } \\
\text { - Image Compression } \\
\text { - Finding patterns in high } \\
\text { dimension data }\end{array}$ & $\begin{array}{l}\text { - PCA is tool, In Image Fusion } \\
\text { having property of transforming } \\
\text { the correlated variables into } \\
\text { uncorrelated variables. }\end{array}$ & $\begin{array}{l}\text { - May produce spectral } \\
\text { degradation. }\end{array}$ \\
\hline 3. & $\begin{array}{l}\text { IHS(intensity, } \\
\text { Hue, Saturation } \\
\text { ) [12] }\end{array}$ & $\begin{array}{l}\text { - Used for Geologic } \\
\text { mapping. }\end{array}$ & $\begin{array}{l}\text { - RGB changes into IHS Easily } \\
\text { used for Image sharpening. } \\
\text { - Resolution increases, improves } \\
\text { image intensity and enhance it } \\
\text { more. }\end{array}$ & $\begin{array}{l}\text { - Can't decompose image } \\
\text { into frequencies. } \\
\text { - Color distortion is often } \\
\text { significant. }\end{array}$ \\
\hline 4. & $\begin{array}{l}\text { ICA(Independe } \\
\text { nt Component } \\
\text { Analysis)[2] }\end{array}$ & $\begin{array}{l}\text { - Estimated transform can be } \\
\text { applied to application field. }\end{array}$ & - More degree of freedom. & - Not shif \\
\hline 5. & $\begin{array}{l}\text { Average } \\
\text { Method[12] }\end{array}$ & $\begin{array}{l}\text { - It is used where all regions } \\
\text { of images are in focus. } \\
\text { Selects regions where } \\
\text { higher pixel values found. }\end{array}$ & $\begin{array}{l}\text { - Easiest and best method of } \\
\text { image fusion. } \\
\text { - fast running speed is main } \\
\text { advantage. }\end{array}$ & $\begin{array}{l}\text { - In pixel level method not } \\
\text { sure to have a clear objects } \\
\text { from the set of images. }\end{array}$ \\
\hline 6. & $\begin{array}{l}\text { Maximum } \\
\text { Method[12] }\end{array}$ & & $\begin{array}{l}\text { - Resulting in highly focused } \\
\text { image output depending on the } \\
\text { input images. }\end{array}$ & $\begin{array}{l}\text { - It is a method that } \\
\text { affected by blurring effect } \\
\text { which directly harm on } \\
\text { the contrast of the image. }\end{array}$ \\
\hline 7. & $D W T[17]$ & & $\begin{array}{l}\text { - It provides better signal to noise } \\
\text { ratio than pixel based approach. } \\
\text { different rules are applied } \\
\text { depending on their frequencies( } \\
\text { low or high) }\end{array}$ & $\begin{array}{l}\text { - Less spatial resolution } \\
\text { and can't fuse images of } \\
\text { different sizes. }\end{array}$ \\
\hline 8. & $\begin{array}{l}\text { Minimum } \\
\text { Method[12] }\end{array}$ & & $\begin{array}{l}\text { - Used in case of darker images } \\
\text { where darkness relates to noise. }\end{array}$ & $\begin{array}{l}\text { - The only perception is } \\
\text { that it selects those pixels } \\
\text { having minimum intensity } \\
\text { and avoid other pixels. }\end{array}$ \\
\hline
\end{tabular}

The various methods have its advantages and disadvantages in various domains with the main motive of combining two images to provide a good quality image.

\section{EXISTING APPROACHES FOR IMAGE FUSION TECHNIQUES}

The various researchers and authors tried their level to use the existing technique of image fusion for providing good quality images. In their approaches various methods/algorithms are combined to produce better images. The description for the techniques used, are discussed in Table II with its key benefits. It is found that the researchers provided the best approaches for producing the desired results but there are still some improvements that should be focused, needed for their existing proposed techniques. The various limitations are also discussed as research gaps in TableII. 
Table II. Discussion on Existing Approaches suggested by various Authors

\begin{tabular}{|c|c|c|c|c|}
\hline S.NO & Author & Techniques used & Benefits & Gaps \\
\hline 1. & $\begin{array}{l}\text { Pandit et } \quad \text { al. } \\
(2015) \text { [12] }\end{array}$ & $\begin{array}{l}\text { Evaluation of Image } \\
\text { Fusion algorithms in } R S\end{array}$ & $\begin{array}{llr}\text { - } & \text { Acquires more } & \text { accuracy } \\
\text { Elimination of } & \text { redudancy } \\
\text { provides reliable image data. }\end{array}$ & $\begin{array}{l}\text { - Color distortion is still } \\
\text { a significant problem. }\end{array}$ \\
\hline 2. & $\begin{array}{l}\text { Ejaily et al. } \\
(2013)[2]\end{array}$ & Improved ICA & $\begin{array}{l}\text { The improved ICA image fusion } \\
\text { method has provided good quality } \\
\text { images with its contrast specific. }\end{array}$ & $\begin{array}{l}\text { - The traditional ICA } \\
\text { methods provides the } \\
\text { images which are not of } \\
\text { good quality as well as } \\
\text { the fused images are } \\
\text { further transform to } \\
\text { IHS color space which } \\
\text { is bit time consuming. }\end{array}$ \\
\hline 3. & $\begin{array}{l}\text { Jin et al. } \\
(2011)[5]\end{array}$ & $\begin{array}{l}\text { Improved IHS transform } \\
\text { helps in using the } \\
\text { panchromatic image } \\
\text { degradation model. }\end{array}$ & $\begin{array}{l}\text { - Feasible and has more efficiency } \\
\text { helps to reduce the spectral } \\
\text { distortion, the resultant image } \\
\text { contains more information and } \\
\text { clear. }\end{array}$ & \\
\hline 4. & $\begin{array}{l}\text { Prakash et al. } \\
\text { (2013)[13] }\end{array}$ & $\begin{array}{l}\text { Biorthogonal } r \text { Wavelet } \\
\text { Transform (BWT) using } \\
\text { Maximum rule }\end{array}$ & $\begin{array}{l}\text { - (WT) based method makes fused } \\
\text { images better by reducing loss of } \\
\text { valuable information and } \\
\text { distortions produces by the spatial } \\
\text { domain techniques. } \\
\text { - Linear phase and symmetry } \\
\text { properties helps in retaining } \\
\text { information of images like lines, } \\
\text { egdes, boundaries curves. }\end{array}$ & $\begin{array}{l}\text { - } \text { Brovery transform is } \\
\text { limited to three bands } \\
\text { and the multiplicative } \\
\text { methods introduce } \\
\text { distortions. }\end{array}$ \\
\hline 5. & $\begin{array}{l}\text { Liu et al. } \\
(2013)[9]\end{array}$ & $\begin{array}{l}\text { Multifocus image fusion } \\
\text { by using lifting scheme of } \\
\text { Wavelets }\end{array}$ & $\begin{array}{ll}\text { - } & \text { Fastest computational speed takes } \\
\text { less memory easier to implement. } \\
\text { - Focuses on high frequency } \\
\text { details of images. }\end{array}$ & $\bullet$ \\
\hline 6. & $\begin{array}{l}\text { Sharmila et al. } \\
\text { (2013)[6] }\end{array}$ & $\begin{array}{l}\text { Discrete wavelet transform } \\
(D W T) \text { with Entropy } \\
\text { concepts }\end{array}$ & 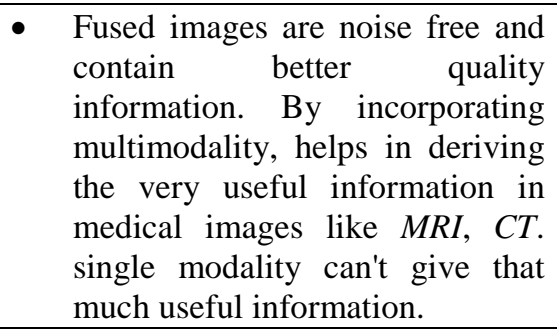 & $\bullet$ \\
\hline 7. & $\begin{array}{l}\text { Kaur et al. } \\
\text { (2015) [8] }\end{array}$ & $P C A$ & $\begin{array}{l}\text { - A large number of input } \\
\text { information can be compressed } \\
\text { into small amount without any } \\
\text { loss of information. } \\
\text { - } \text { removes redundancy. } \\
\text { combination of DWT with PCA } \\
\text { improves the performance. }\end{array}$ & $\begin{array}{l}\text { - } \text { may not be satisfied to } \\
\text { fuse high and low } \\
\text { resolution } \\
\text { multispectral(MS) } \\
\text { images. }\end{array}$ \\
\hline
\end{tabular}

From Table II, it is concluded that Pandit et al. [12] uses the technique based on the picture element i.e pixel has shown the comparatievely more effective and requires sufficient time for implementation. Hence the performance is compared. Ejaily et al. [2] discussed, the Independent Component Analysis based image fusion technique gives efficient fusion results by providing eminent values for the pixel based resolution and component based resolution. Prakash et al [13] defined the a fusion method which is based on pixel resolution. The advantage of this technique is that, it efficiently defends all the important details of the boundary, while undergoing the fusion process. Sehasnainjot et al [15]. real firmed that the maximum rule of fusion i.e rule that merges the infinitely large pixel values from images for fusion, gives better results, when analyzed quantitatively and qualitatively on the real time fingerprint images. K Sharmilla et al. [6] discussed the image fusion of medical images. This particular technique allows to consolidate the images registered with different scanners (multimodality) into a individual fused image. Thus it is found that all the existing techniques of image fusion helps in producing 
quality images but due to their limitation or provisions to various application domains, these techniques have their characteristics or specified outputs accordingly. For example

\section{CONCLUSION}

In this paper, the comparative study of spatial domain and transform domain image fusion techniques are provided and also a discussion about the use of image fusion techniques in remote sensing applications. Although selection of fusion algorithm is problem dependent but this review results that the spatial domain techniques have image blurring problem. To overcome

\section{VI.REFERENCES}

[1] Digital Globe (2003), QuickBird scene 000000185959_01_P005, Level Standard 2A, Digital Globe, Longmont, Colorado, 2003

[2] A. E. Ejaily, M. Y. E. Nahas, and G.Ismail. "A New image fusion technique to improve the quality of remote sensing images," International Journal of computer Science Issues (IJCSI), Vol.10, Issue 1, (january 2013) pp. 565-569

[3] C. Pohl and J. L. V. Genderen. "Multisensor Image Fusion in Remote Sensing: Concepts, Methods, and Applications," International journal of Remote Sensing , (Nov 2010)Vol.19, pp. 823-854.

[4] Ghassemian and Hassan, "A Review of Remote Sensing Image Fusion Methods." Information Fusion ELSEVIER, Vol. 32, (2016) pp 75-89.

[5] Jin, Baoxuan and S. Li, "Applications of Remote Sensing Data Fusion Technology Based on Improved IHS Transform in the ASEAN Geospatial Public Service Platform," IEEE. 2011 pp 1-4.

Image and Data Fusion (ISDIF) International Symposium

[6] K. Sharmila, S. Rajkumar and V. Vijayarajan, "Hybrid Method for Multimodality Medical Image Fusion using Discrete Wavelet Transform and Entropy concepts with Quantitative Analysis," International Conference on Communication and Signal Processing. INDIA: IEEE, April 2013 pp 489-493.

[7] R. KAUR, "Review on Image Fusion and its techniques." IRACST International Journal of Computer Networks and Wireless Communications (IJCNWC), April 2015.

[8] V. Kaur and J. Kaur, "Comparison of Image Fusion Techniques: Spatial and Transform Domain based Techniques." International Journal Of Engineering And Computer Science ISSN:2319-7242, May 2015, pp. 12109-12112.

[9] Liu, Lixin, H. Bian, and G.shao, "An Effective Wavelet based scheme for multi-focus image fusion." International Conference on Mechatronics and Automation. japan: IEEE, 2013 pp. 1720-1725.
IHS and ICA do not provide colored images but the approaches are best suited for medical images due to its efficiency.

the disadvantages of these methods, frequency domain based methods can be used or can be combined with spatial domain methods. The wavelet transforms is the very good technique for the image fusion providing high quality spectral content. But a best fused image is of qualities of both images of the integration of DWT and spatial domain fusion method fusion algorithm enhance the througput. Finally, this review concludes that image fusion algorithm based on combination of PCA with DWT will improve the image fusion quality to visualize the hidden information clearly.

[10] Mahajan, Shveta, and A. Singh, "A Comparative Analysis of Different Image Fusion Techniques." International journal of Computer Science (IJCS) Vol. 2, no. 1 (2014).

[11] Mandhare, R. Ashok and P. Upadhyay, "Pixel-level Image fusion using Brovey Transform and Wavelet Transform." International Journal of Advanced Research in Electrical, Electronics and Instrumentation Engineering, June 2013 Vol. 2, no. 6, pp. 2690 - 2695.

[12] Pandit, vaibhav R., and R. j. Bhiwani, "Image fusion in Remote Sensing Applications: A review, "International Journal of Computer Applications (june2015) Vol. 120 No. 10.

[13] Prakash, Om, R. Srivastava, and Ashish Khare, "Biorthogonal Wavelet Transform Based Image Fusion Using Absolute Maximum Fusion Rule." Conference on Information and Communication Technologies (ICT 2013). Proceedings of 2013 IEEE, 2013 pp. 577-582.

[14] Sahu, D. Kumar, "Different Image Fusion Techniques," International Journal of Modern Engineering Research (2012) Vol. 2 No. 5 pp. 4298-4301.

[15] S. Singh and N. Neeru, "Finger Print Fusion Using Daubechies (Db1)Wavelet Transformation and quality measures." 2nd International Conference on Computing for Sustainable Global Development. New Delhi (India), Marc, 2015 pp. 822-826.

[16] Thomas, Elizabeth, P. B. Nair, S. N. john, and M. Domiic,"ImageFusion using Daubechies Complex Wavelet Transform and Lifting wavelet Transform: a Multiresolution Approach." International Conference on Magnetics \& Drives (AICERA-1014 iCMMD). IEEE, 2014 pp. 1-5.

[17] S. S. Malik, S. P. P. Kumar, and G. B. Maruthi,"DT-CWT: Feature level image fusion based on dual-tree complex wavelet transform," in International Conference on Information Communication and Embedded Systems (ICICES), pp. 1-7, Feb 2014. 